\title{
La Educación Ambiental en las redes sociales: \#EA26
}

\author{
Sonia Calvo Rubio \\ Easempervirens.EAsempervirens@gmail.com \\ ORCID: https://orcid.org/0000-0001-6033-3067 \\ Josechu Ferreras Tomé \\ Argos, Proyectos educativos. josechuferreras@educa.org \\ ORCID: https://orcid.org/0000-0001-6964-7222 \\ Daniel Rodrigo-Cano \\ Universidad de Sevilla.drodrigo1@us.es \\ ORCID: https://orcid.org/0000-0003-2753-5470
}

[Recibido: 16 Julio 2019. Revisado: 27 Noviembre 2019. Aceptado: 2 Febrero 2020]

\begin{abstract}
Resumen: La facilidad de uso de las redes sociales las han convertido en una excelente herramienta para dar a conocer las ideas, transmitir conocimiento y establecer debates. En esta investigación se pone de manifiesto el caso de \#EA26 como ejemplo de un buen uso de la red social Twitter para mantener debates y encuentros virtuales a través de una experiencia singular y que ha supuesto un auténtico impacto, logrando la puesta en valor de la Educación Ambiental como herramienta necesaria frenar la crisis ambiental y para alcanzar la transición ecológica. En este artículo se realiza una descripción del funcionamiento, los logros y la situación actual de la Educación Ambiental en las redes sociales y una evaluación cualitativa del proceso seguido y los retos que tiene por delante \#EA26 como caso de estudio. Se ha planteado una entrevista a las dieciséis personas dinamizadoras del movimiento (ciberactivistas), que ha permitido analizar sus claves, así como lo que ha supuesto y los retos a los que se enfrenta. De la misma forma, se ha analizado una perspectiva de futuro. Con estas herramientas se concluye que \#EA26 se ha consolidado como un referente para la Educación Ambiental en las redes sociales y que tiene el reto de ser referente en otros ámbitos.
\end{abstract}

Palabras clave: Educación Ambiental, Redes sociales, Ciberactivismo. Comunicación ambiental. Educomunicación.

\section{Environmental Education in social networks: \#EA26}

Abstract: The ease of use of social networks has made them an excellent tool to make ideas known, transmit knowledge and establish debates. This research highlights the case of \# EA26 as an example of a good use of the social network Twitter to play debates and virtual meetings through a unique experience and that has been a real impact by making the value of Education Environmental as a necessary tool to stop the environmental crisis and to achieve the ecological transition. In this article, a description of the functioning, achievements and current status of Environmental Education in social networks is presented, as well as a qualitative evaluation of the process followed and the challenges that lie ahead. \# EA26 as a case study. An interview has been proposed to the sixteen dynamic people of the movement, who could be considered as cyberactivist, who have allowed to analyze the keys of the same, as well as what it has supposed and the challenges it faces, in the same way, has analyzed a future perspective. With these tools it is concluded that \# EA26 has consolidated as a reference for Environmental Education in social networks and that it has the challenge of being a reference in other areas.

Keywords: Environmental Education, Social Networks, Cyberactivism

Para citar este artículo: Calvo, S., Ferreras, J., y Rodrigo-Cano, D.(2020) La Educación Ambiental en las redes sociales: \#EA26. Revista de Educación Ambiental y Sostenibilidad 2(1), 1301. doi: 10.25267/Rev_educ_ambient_sostenibilidad.2020.v2.i1.1301 


\section{Introducción}

La Educación Ambiental es entendida como un ámbito de pensamiento y acción en el que predomina la heterogeneidad y el debate (García, 2002). Este concepto ha evolucionado en el tiempo: así, mientras Breiting (1985) lo define como la educación que, de formas diversas, trata la relación problemática del ser humano con la naturaleza incluyendo la degradación ambiental, la explotación y el reparto de los recursos, el crecimiento de la población humana y el exterminio de las especies animales y vegetales con el fin de lograr personas activas y bien informadas. En la actualidad se puede llegar a entender que la Educación Ambiental como una corriente internacional de pensamiento y acción cuya meta es procurar cambios individuales y sociales que provoquen la mejora ambiental y un desarrollo sostenible (Ministerio de Agricultura, Pesca, Alimentación y Medio Ambiente, 1999).

En todo caso, estas características parecen incomodar al sistema neoliberal, que la considera como un arma para manipular la mente de las personas y es especialmente significativo en el tratamiento pedagógico del cambio climático que es aprovechado para politizar desde enfoques negacionistas o ultra-neo-liberales (Meira, 2002) y de paso ultra-precarizar el sector de la Educación Ambiental (Meira, Barba, y Lorenzo, 2017).

El colectivo \#EA26 toma como referencia el Libro Blanco de la Educación Ambiental en España, y entiende la Educación Ambiental en un sentido amplio, que incluye sensibilización, comunicación, participación y la propia educación ambiental (Ferreras, Calvo y Rodrigo-Cano, 2019). Para ello se han de tener en cuenta problemas ambientales en la perspectiva de cambiar el estilo de vida y el modelo de desarrollo consumista en el que vivimos (Piñeiro y Díaz-González, 2019). De la misma forma, el colectivo pretende dirigir sus actuaciones a toda la población y a todos los sectores sociales y tiene especial importancia para alcanzar los Objetivos de Desarrollo Sostenible (Rodrigo-Cano, Picó y Di Muro, 2019).

Ante esta circunstancia es necesario potenciar a los "educandos como emisores, ofreciéndoles posibilidades, estímulos y capacidades para la autogeneración de mensajes" (Kaplún, 1998: 244), de esta forma tanto el educador como el profesional de los medios se convierten en mediadores en el proceso de comunicación educativa como base para una educomunicación ambiental (Rodrigo-Cano, 2018).

Ante esta situación de educomunicación ambiental y de emergencia climática es necesario establecer procesos comunicativos que promuevan la participación cívica y el diálogo a través de una información completa, veraz y contrastada (Meira, 2013). Por tanto, es necesario que la educación y la comunicación se orienten al desarrollo de procedimientos para involucrar directamente a la ciudadanía en el desarrollo de políticas (Brulle, 2010).

\section{La RED y la EA}

La interrelación de las TIC y la Educación Ambiental está aumentando en los últimos años. Para Cabero y Llorente (2005) las TIC más utilizadas son: el vídeo como transmisor de información y el vídeo como instrumento de conocimiento (herramientas multimedia como los CD Rom y los DVD); Internet: Webs informativas (Buscadores, Revistas) y formación virtual, desde los formatos más habituales a otros 
más innovadores como los Cursos Masivos On line y Abiertos (MOOC). A esta clasificación deberíamos incluir otras herramientas on line como: Youtube, las redes sociales, Skype y Videojuegos (Ferreras, Calvo y Rodrigo-Cano, 2019).

Las redes sociales se han hecho muy populares. En enero de 2018 Facebook cuenta con cerca de 2.167 millones de usuarios activos en un mes (Kemp, 2018); esta popularidad se debe, en parte, al uso y desarrollo de la telefonía móvil. En 2017 había 7.740 millones de líneas de telefonía móvil (más que habitantes) y 3.475 millones de usuarios de internet en el mundo (Fundación Telefónica, 2017), mientras que en España el 91,7\% de los usuarios de la telefonía móvil lo utiliza para conectarse a Internet (Fundación Telefónica, 2017).

Es habitual que organizaciones ambientales como Greenpeace o sociales como Amnistía Internacional utilicen las redes sociales para la difusión y coordinación de sus acciones y campañas (Castillo, 2014; Vázquez, 2015). Esta habitualidad en el uso de las redes sociales permite crear comunidades de aprendizaje alrededor de ellas (Aznar y Martínez, 2013). Por tanto, podríamos afirmar que las redes sociales posibilitan las acciones proambientales entre individuos y grupos sociales para lograr una acción informada y decidida a favorecer el entorno y a construir una sociedad consciente, tal como pretende el Libro Blanco de la Educación Ambiental (Ministerio de Agricultura, Pesca, Alimentación y Medio Ambiente, 1999).

En el Congreso Nacional de Medio Ambiente del año 2010 (CONAMA10) se realizó una sesión específica de redes sociales titulada Comunicación 2.0, que fue coordinada por Roberto Ruiz de la Asociación de Ciencias Ambientales, donde se puso en valor el creciente interés por las redes sociales. Como resultado se editó la guía "Cómo usar las redes sociales para actuar en el medio ambiente" donde se da importancia a cómo desde la Web 2.0 se están desarrollando tecnologías que posibilitan la comunicación, la educación y la participación proambientales (Asociación de Ciencias Ambientales, 2010).

Así, Ojeda-Barceló, Gutiérrez-Pérez y Perales-Palacios (2009) recogen los usos más habituales que realizan educadoras y educadores ambientales de Internet: comunicación directa, adquisición de información y recursos, mediante la evaluación de páginas web relacionadas con Educación Ambiental. También hacen propuestas en las que se plantea Internet como laboratorio científico remoto, se promueve explorar nuevos campos en educación abierta a distancia (Open Distance Learning, ODL, el elearning), el uso de Internet como fuente de recursos y recopilaciones, así como trabajos para favorecer el manejo de Internet y la búsqueda de recursos, formación de docentes y educadores y educadoras ambientales, propuestas de entornos colaborativos aplicados a la Educación Ambiental, construcción de entornos virtuales y modelos de simulación en los que se muestran procesos o problemas complejos de una forma sencilla y visual $y$, finalmente, trabajos concretos sobre programas que hacen uso de TIC.

\section{El caso de \#EA26}

La iniciativa \#EA26 surge de la interacción de tres personas por la red social Twitter, en las que se comenta la escasa presencia de la Educación Ambiental en las redes sociales. Por ello, se proponen convocar un evento que le dé más visibilidad para el Día Mundial de la Educación Ambiental, el 26 de enero de 2014. A partir de esta 
conexión, se crea una cuenta de Gmail, otra en Twitter y se diseña un blog que da soporte la propuesta.

\#EA26 se define como un punto de encuentro entre educadores y educadoras ambientales en las redes sociales que surge de y desde las redes sociales (Benayas, Marcén, Alba y Gutiérrez, 2017), inicialmente desde un blog en Wordpress denominado \#EA26 Educación Ambiental, y desde la red social Twitter en la cuenta @edu_ambiental (Figura 1). Más tarde también está presente en otras redes sociales como Facebook e Instagram.

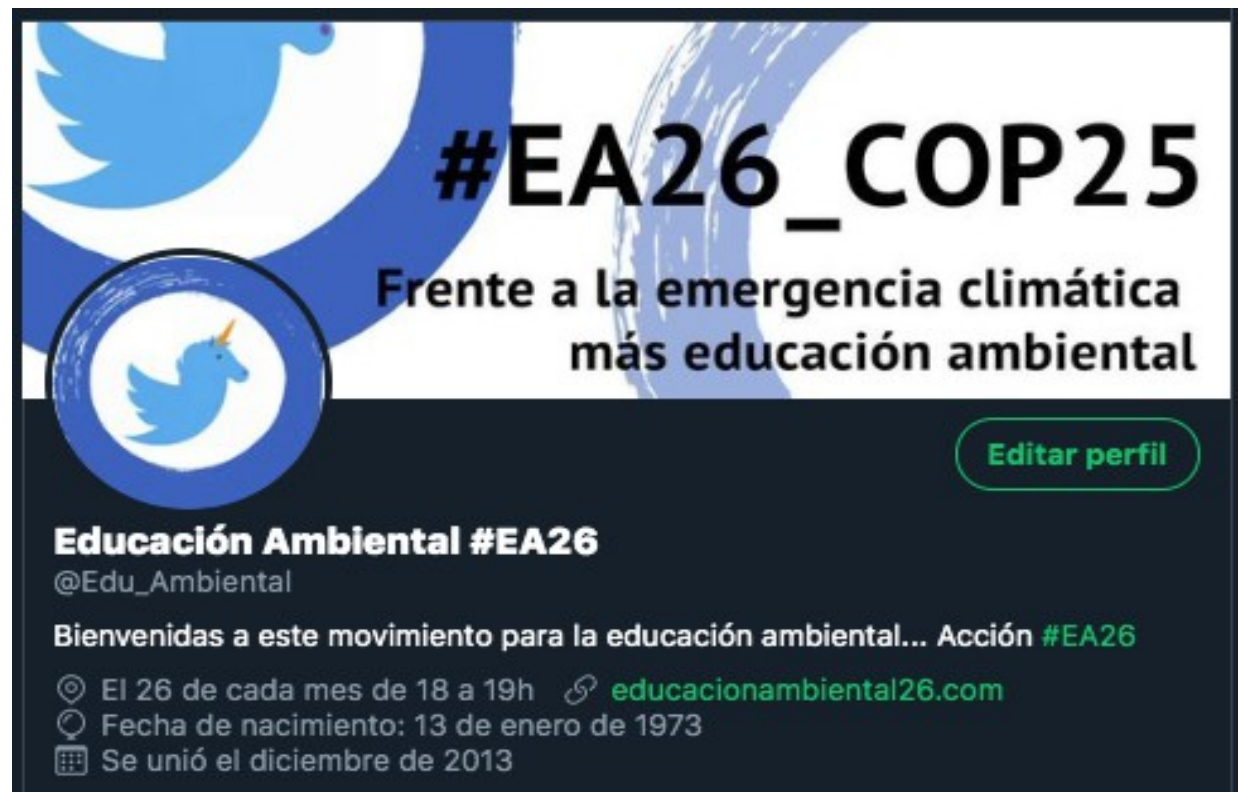

Figura 1. Imagen de Twitter de \#EA26.

Todos estos espacios se gestionan de forma colaborativa a través de la cogestión o a través de invitación para la creación de contenidos y la participación en los debates. Por tanto, el número de personas del grupo dinamizador ha ido aumentando de forma habitual y constante, pasando de 3 a 7 en un primer momento y llegando hasta las 16 personas que lo componen en la actualidad. De esta manera se generan nuevos impulsos, iniciativas y enriquecimiento en las relaciones, en los puntos de vista y en nuevas acciones.

Todas las personas integrantes de este movimiento parten del voluntariado, la gestión colaborativa y del considerar que las redes permiten mayor presencia pública $y$, de esta forma, dan a conocer las posibilidades, opciones, temas, reflexiones, citas de interés en torno a la educación ambiental en los términos que recoge el Libro Blanco de la Educación Ambiental (Ministerio de Agricultura, Pesca, Alimentación y Medio Ambiente, 1999): "promover la acción pro-ambiental entre individuos y grupos sociales; una acción informada y decidida a favor del entorno y hacia una sociedad consciente, realizada en el contexto vital de los ciudadanos: hogar, trabajo, escuela, ocio y comunidad" (Navarro et al., 2016).

A fin de cuentas, \#EA26, como indica Ruiz (2015: 5), se trata de una "iniciativa que consiste en que el día 26 de cada mes entre las 18 y las 19 horas se utiliza la etiqueta \#EA26 para compartir reflexiones, experiencias, proyectos de futuro relacionados 
con la comunicación, sensibilización y participación en torno a la educación ambiental".

Desde 2014 en \#EA26 ha habido 49 debates y 18 acciones con personas invitadas con relevancia en el ámbito de la Educación Ambiental, que han tratado una amplia diversidad de temas y han permitido conocer la relevancia que tienen en las redes los asuntos de sostenibilidad (De la Osa, 2018). Se han categorizado las temáticas de los debates siguiendo los marcos de acción del Libro Blanco de la Educación Ambiental, pero, dado que la situación social y ambiental ha evolucionado en estos 20 años desde su publicación, se ha realizado una segunda categorización por temáticas (Tablas 1 y 2).

Tabla 1. Temas tratados en debates \#EA26

Marcos de Acción Libro Blanco de la Educación Ambiental

Temática Tratada

Totales de Marcos de

Administración general y autonómica

Política
Acción

\begin{tabular}{|c|c|c|}
\hline & Política & 4 \\
\hline \multirow[t]{3}{*}{ Comunidad: Asociaciones } & & 2 \\
\hline & Intervención Social & 1 \\
\hline & Voluntariado & 1 \\
\hline \multirow[t]{6}{*}{ Comunidad: Ciudadanía } & & 8 \\
\hline & Consumo & 4 \\
\hline & Incendios & 1 \\
\hline & Intervención Social & 1 \\
\hline & Medio rural & 1 \\
\hline & Turismo & 1 \\
\hline \multirow[t]{6}{*}{ Empresas de Educación Ambiental } & & 7 \\
\hline & Custodia del territorio & 1 \\
\hline & Equipamientos & 1 \\
\hline & Espacios Naturales & 1 \\
\hline & Estrategias & 1 \\
\hline & Metodologías & 3 \\
\hline \multirow[t]{3}{*}{ Empresas En general } & & 2 \\
\hline & Empresa & 1 \\
\hline & Política & 1 \\
\hline \multirow[t]{3}{*}{ Medios Comunicación } & & 2 \\
\hline & Comunicación & 1 \\
\hline & Cine & 1 \\
\hline \multirow[t]{2}{*}{ Sistema educativo Infantil, Primaria y Secundaria } & & 1 \\
\hline & Equipamientos & 1 \\
\hline \multirow[t]{2}{*}{ Sistema educativo Universitaria } & & 1 \\
\hline & Sistema educativo formal & 1 \\
\hline \multirow[t]{2}{*}{ Sistema educativo } & & 1 \\
\hline & Sistema educativo formal & 1 \\
\hline \multirow[t]{7}{*}{ Otros } & & 21 \\
\hline & Cambio Climático & 4 \\
\hline & Género & 1 \\
\hline & Impactos & 2 \\
\hline & $\begin{array}{l}\text { Objetivos de Desarrollo } \\
\text { Sostenible }\end{array}$ & 1 \\
\hline & Profesionalización & 2 \\
\hline & Retos & 11 \\
\hline
\end{tabular}




\begin{tabular}{|c|c|c|}
\hline Total temas tratados & & 49 \\
\hline \multicolumn{3}{|l|}{ Tabla 2. Temas tratados en \#EA26_Con } \\
\hline $\begin{array}{l}\text { Marcos de Acción Libro Blanco de la } \\
\text { Educación Ambiental }\end{array}$ & Temática Tratada & $\begin{array}{c}\text { Totales de Marcos de } \\
\text { Acción }\end{array}$ \\
\hline \multirow[t]{2}{*}{ Administración general y autonómica } & & 3 \\
\hline & Cambio Climático & 3 \\
\hline \multirow[t]{3}{*}{ Comunidad: Administración local } & & 2 \\
\hline & Energía & 1 \\
\hline & Salud & 1 \\
\hline \multirow[t]{4}{*}{ Comunidad: Ciudadanía } & & 4 \\
\hline & Consumo & 2 \\
\hline & Ecofeminismo & 1 \\
\hline & Economía & 1 \\
\hline \multirow[t]{2}{*}{ Empresas De Educación Ambiental } & & 2 \\
\hline & Profesionalización & 2 \\
\hline \multirow[t]{3}{*}{ Medios Comunicación } & & 5 \\
\hline & Paisaje & 1 \\
\hline & Otro & 4 \\
\hline $\begin{array}{l}\text { Sistema educativo Infantil, Primaria y } \\
\text { Secundaria }\end{array}$ & & 1 \\
\hline \multirow[t]{2}{*}{ Sistema educativo Universitaria } & & 1 \\
\hline & Profesionalización & 1 \\
\hline Total general & & 18 \\
\hline
\end{tabular}

La recopilación de los tuits de estos debates está disponible para su consulta o análisis en la página web en formato PDF o de texto.

A partir de este primer hito, se podrían señalar otros significativos en esta experiencia:

1) Organización del I Encuentro Presencial. Tras múltiples peticiones en los debates, de forma autofinanciada se organiza el I Encuentro Presencial de \#EA26 en Madrid, en el que participan 60 personas de distintas comunidades autónomas. Este Encuentro permite a \#EA26 consolidarse, darse visibilidad interna y externa como colectivo, compartir y matizar opiniones e impulsar la presencia de \#EA26 en otros congresos, jornadas, cursos y encuentros.

2) Reunión con la Ministra de Transición Ecológica. Tras un contacto en Twitter con la Ministra de Transición Ecológica, se propone una reunión la siguiente semana. A través de WhatsApp se define el grupo de personas que van a la reunión y paralelamente en Drive se va construyendo un documento con las aportaciones de todo el equipo de \#EA26, que se resumen en un decálogo que se presenta a la ministra el día de la reunión (Figura 2).

El movimiento \#EA26 aprovecha que la Web 2.0 se ha convertido en una potente herramienta para facilitar la participación conectando a personas con objetivos comunes, y, además, que las redes sociales posibilitan la difusión de la información, especialmente a través de la imagen (León y Bourk, 2018) y la asociación de las redes sociales visuales como Youtube, Twitter, Facebook e Instagram proporcionan información a la ciudadanía sobre temáticas de interés entre las que se encuentran temas como ciencia y tecnología. Las redes sociales (Facebook y Twitter) y los vídeos (Youtube) son las principales fuentes de información entre los grupos de edad de 15 a 
54 años, según la Encuesta de Percepción Social de la Ciencia y la Tecnología en España (Revuelta y Corchero, 2017).

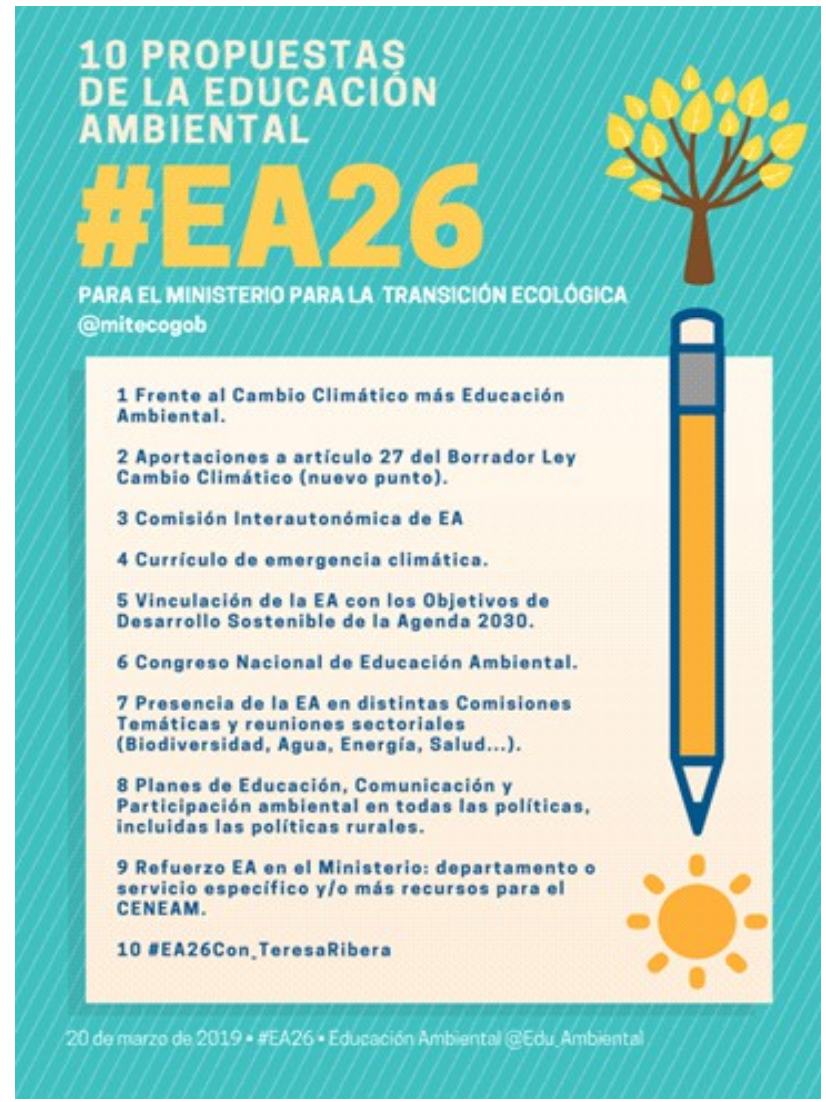

Figura 2. Propuestas de Educación Ambiental presentadas a la Ministra de Transición Ecológica.

Las acciones de información buscan sensibilizar-movilizar-actuar-protestar y, según su origen, existen acciones espontáneas como resultado de un acontecimiento que va cobrando importancia en las redes hasta lograr relevancia generando conversación y llegar a ser tendencia -trending topic-, a través de utilizar etiquetas (hashtags) (Ruiz, 2015). Este es el caso de \#EA26 que, en varias ocasiones, en sus debates y encuentros ha sido trending topic (Figuras 3 y 4 ) a través de tuits de opinión-queja-propuesta (Toboso, 2016; Toboso, de Casas y Rodrigo-Cano, 2018).

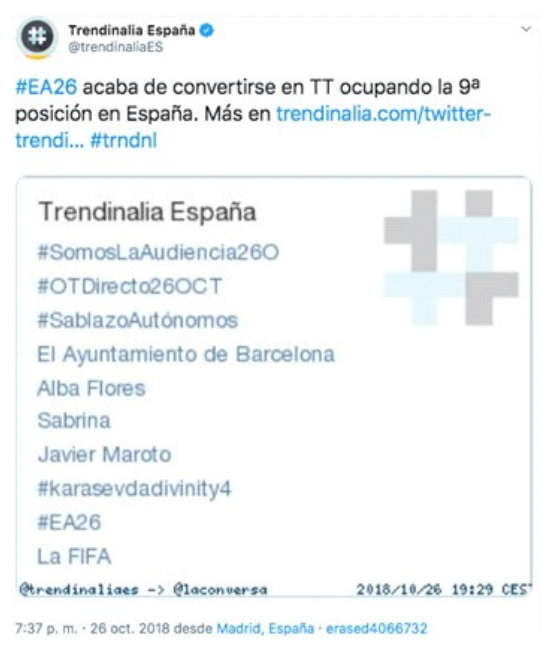

Figura 3. Imagen de Twitter de \#EA26 siendo Trendig Topic el 26 de octubre de 2018. 


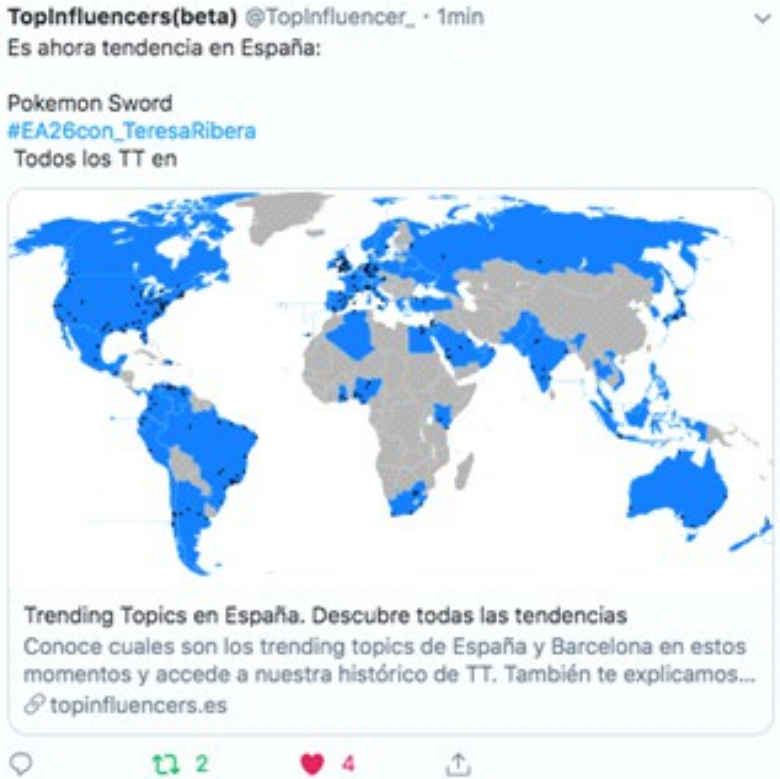

Figura 4. Imagen de Twitter de \#EA26Con_TeresaRibera siendo Trendig Topic el día en el que participó la Ministra (en funciones) de Transición Ecológica.

Además, \#EA26 participa de forma habitual en diferentes redes sociales como Twitter con más de 4.600 seguidores en el mes de Diciembre de 2019. Desde 2014, ha tuiteado 10.700 mensajes. En Facebook, la página tiene 533 seguidores y en Instagram 237 seguidores, mientras que en el blog educacionambiental26.com tiene 288 seguidores y más de 60.000 visitas, que han aumentado más del doble entre 2015 (5.881 visitas) y 2018 con 14.329 visitas, siendo la página principal la más visitada con prácticamente 22.000 visitas.

\section{Metodología}

En este trabajo se ha realizado, por una parte, una descripción de las dinámicas propias de \#EA26, y por otra parte, utilizando una metodología cualitativa, se han realizado 16 entrevistas on line a las personas dinamizadoras del movimiento, con un formato de entrevista semi-estructurada. La entrevista está dirigida para conocer las opiniones, las actitudes y motivaciones de los mismas respecto al propio \#EA26.

Las entrevistas se han realizado en formato virtual a través de google forms porque posibilita el acceso directo tanto para las personas entrevistadas como para las entrevistadoras. Se realizaron cuatro preguntas:

1.- ¿Cuáles crees que son las claves de \#EA26? Propuestas de mejora;

2.- ¿Qué ha supuesto \#EA26 para la educación ambiental?;

3.- ¿A qué retos se enfrenta \#EA26 en la actualidad?;

4.- ¿Qué horizonte definirías para \#EA26?

Tras la definición de las preguntas se analizaron siguiendo un libro de códigos y categorías para poder obtener resultados. 


\section{Resultados}

Tras el análisis de las respuestas se elaboraron los resultados. Estos señalan, en síntesis, cuatro puntos que permiten conocer la opinión de las personas dinamizadoras del movimiento \#EA26, identificar las claves de funcionamiento y las tendencias de futuro a las que se puede dirigir:

\section{¿Cuáles crees que son las claves de \#EA26? Propuestas de mejora.}

Las respuestas recibidas en este apartado han sido muy diversas y los podemos clasificar (Figura 5):

1. Respecto al funcionamiento: destaca la horizontalidad, la descentralización en red en función de liderazgos flexibles y compartidos; la generosidad que nace del compromiso voluntario con la Educación Ambiental de las personas participantes; el espíritu crítico, abierto y respetuoso en los debates; la agilidad en dar respuesta y el estar en continua evolución con una actitud propositiva.

2. Respecto al papel que está cumpliendo \#EA26 se destaca: ser un novedoso espacio de participación, un nexo que aglutina a distintos sectores, que se ha convertido en referente de la Educación Ambiental. Esto puede tener influencia a la hora de hacer una revisión crítica de la Educación Ambiental, recoger algunas de las exigencias del sector como la profesionalización o la desinstitucionalización, elaborar documentos o proponer actuaciones para desarrollar políticas.

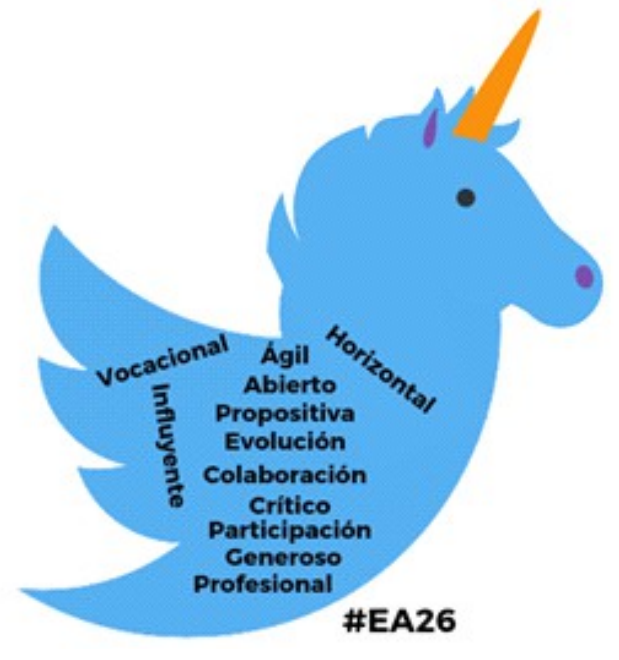

Figura 5. Palabras significativas a la pregunta ¿Cuáles crees que son las claves de \#EA26?

\section{¿Qué ha supuesto \#EA26 para la educación ambiental?}

Según se deduce de las respuestas obtenidas se pueden organizar en torno a cuatro palabras clave (Figura 6):

1. Visibilidad. La Educación Ambiental se ha hecho visible sobre todo dentro del propio sector y más allá de su espacio virtual.

2. Pertenencia. "Ahora sabemos que somos más de lo que aisladamente pensábamos que éramos", resaltando la importancia de que profesionales de distintos ámbitos se encuentren una vez al mes para hablar de Educación Ambiental ha conseguido en la práctica la transversalidad que esta ha perseguido siempre. 
3. Referente. Ser un espacio de referencia en un momento clave en el que la educación ambiental no gozaba de representatividad. Posee un liderazgo integrador que suma transversal y transparentemente entre agentes diversos para reivindicar la necesidad de profesionalizar el sector.

4. Impacto. Tanto como espacio mediático, como por su planteamiento crítico a la hora de tratar el papel de la Educación Ambiental ante distintos problemas socioambientales.

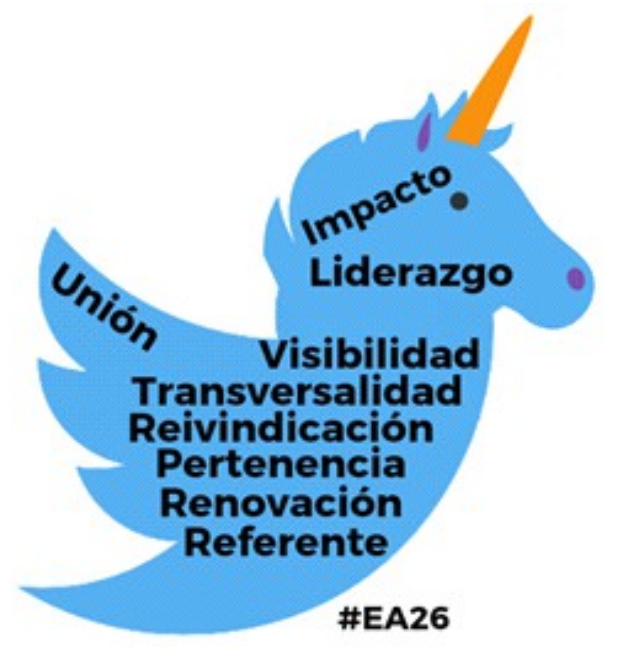

Figura 6. Palabras significativas a la pregunta ¿Qué ha supuesto \#EA26 para la educación ambiental?

\section{¿A qué retos se enfrenta \#EA26 en la actualidad?}

El sumatorio de los retos más importantes que se manifiestan en las respuestas tienen que ver con los retos propios de la Educación Ambiental y otros con los propios del desarrollo de \#EA26 (Figura 7):

1. Retos propios de \#EA26

- Seguir avanzando y evolucionando sin perder la esencia que ha llevado a muchas personas a sumarse a \#EA26.

- Seguir manteniendo el equilibrio entre la espontaneidad y frescura que la caracteriza y la relevancia que está adquiriendo al llegar incluso a las altas esferas políticas.

- Llegar a un público más amplio y conseguir atraer a nuevas personas a los debates. Ser nexo de unión entre la Educación Ambiental y otros grupos de acción. Co-liderar el sector de la Educación Ambiental.

- Pasar de la influencia en las redes sociales a la influencia en la vida real.

- Llegar más allá de las redes sociales. Consolidar lo alcanzado y convertirse en referentes de la Educación Ambiental, siendo capaces de sumar cada vez más esfuerzos.

2. Retos comunes con la Educación Ambiental.

- Apoyar la celebración de un Congreso y la elaboración de una "Estrategia Estatal de Educación Ambiental". 
- Conseguir que las 10 propuestas presentadas a la ministra Teresa Ribera sean una realidad.

- Luchar contra el greenwashing (entendido como amenaza).

- Vinculación de la Educación Ambiental con los Objetivos de Desarrollo Sostenible.

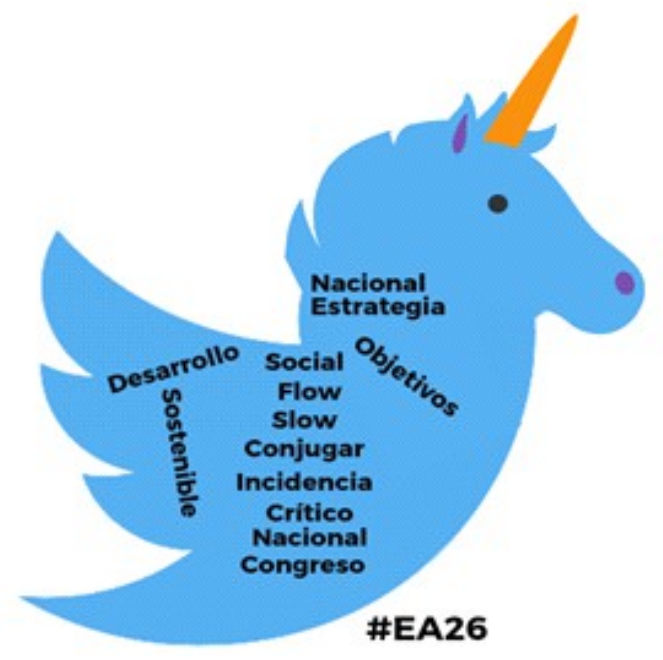

Figura 7. Palabras significativas a la pregunta ¿A qué retos se enfrenta \#EA26 en la actualidad?

\section{¿Qué horizonte definirías para \#EA26?}

El futuro de \#EA26 tras más de cinco años de existencia pasa por mantener su espíritu ciberactivista, utópico e ilusionado. Se plantean tendencias en dos ámbitos: por una parte, respecto al funcionamiento interno y, por otra, en relación a su incidencia social, sin olvidar que la próxima celebración del "II Encuentro presencial de \#EA26" (Figura 8):

Respecto al funcionamiento:

- No definir horizontes, que el grupo motor siga desprendiendo energía y la contagie a todos aquellos que quieran luchar por la Educación Ambiental; que se continúe respondiendo a las demandas externas en la medida de lo que el grupo es sin romper el espíritu de voluntariado o el fluir.

- Continuar en la línea del modelo actual, pero incorporando y escuchando a más colaboradores y colaboradoras con otras visiones para poder abarcar los diferentes proyectos que se vayan presentando en la medida de nuestras posibilidades.

- Debatir sobre diferentes opciones para mejorar la profesionalización, la financiación, etc.

- Analizar y explicitar lo que nos une. Establecer unos criterios/principios comunes que definan e identifiquen EA26 y que sirvan para dilucidar la participación y colaboración en otros ámbitos.

Respecto a su incidencia social: 
- Un \#EA26 que tienda a crear redes con otros sectores y personas para formar esa cámara de resonancia necesaria para alcanzar un cambio real y que el mensaje realmente llegue y se tome en serio.

- En el horizonte 2030 de \#EA26 debe convertirse en un referente más de la Educación Ambiental en España más allá de las redes sociales, participar en movimientos afines, ser una punta de lanza crítica en la lucha contra el cambio climático y por el cambio ecosocial en el camino de la transición ecológica.

- Consolidar \#EA26 como un agente importante a nivel estatal, colaborando en la medida de lo posible para el objetivo común de crear conciencia ambiental, adquirir mayor relevancia y progresivamente constituir un "órgano de consulta" para la Educación Ambiental.

- Continuar e intensificar la acción política, con influencia social y política creciente.

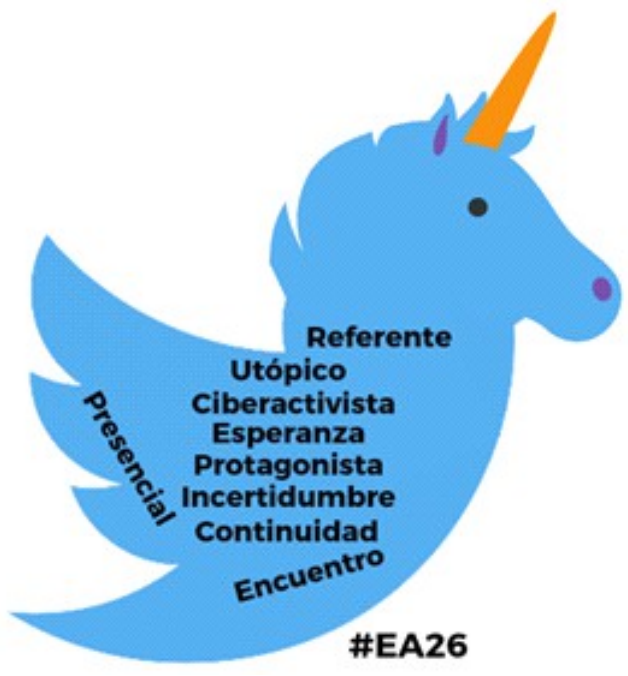

Figura 8. Palabras significativas a la pregunta ¿Qué horizonte definirías para \#EA26?

\section{Conclusiones}

El análisis cuantitativo y categorizado de las temáticas de los debates y las participaciones de las personas en el \#EA26con_ nos permite valorar positivamente las redes sociales como herramienta para acercar el conocimiento científico a través de la Educación Ambiental. Además, el cambio climático se ha convertido en un tema habitual en los medios de comunicación (Fernández-Reyes, 2018) y también en las redes sociales. De esta forma los medios de comunicación son una herramienta fundamental para sensibilizar, movilizar $y$ abrir debates sobre los que ir construyendo el futuro de la Educación Ambiental: ecofeminismo, movilidad, equidad, cambio global, etc. Las redes sociales se han convertido en plataformas indispensables para la distribución y el consumo de informaciones ofreciendo contenidos dependientes de la difusión viral y una capacidad ilimitada de circulación (Alzamora y Braga, 2014).

La primera conclusión del estudio de caso \#EA26 vistos la trayectoria, los datos de participación (número de seguidores y visitas al blog) y la continuidad a lo largo de cinco años, es el cumplimiento del objetivo inicial que se plantearon las personas que 
crearon este espacio de participación: incrementar la presencia y la visibilidad de la Educación Ambiental en las redes sociales, al menos en Twitter, en un momento clave en el que estaba especialmente azotada por la crisis.

Por los mismos motivos \#EA26 también se muestra como una fórmula de éxito en relación a su "forma de hacer" basada en el funcionamiento horizontal en red, liderazgos flexibles y compartidos, el compromiso voluntario con la Educación Ambiental y el grupo, el espíritu crítico y respetuoso en los debates y la agilidad en dar respuestas a las demandas que le llegan.

Del análisis de las respuestas recibidas, se puede afirmar que las personas que forman parte del grupo dinamizador de \#EA26, piensan que se ha convertido en un referente para la Educación Ambiental. Las experiencias profesionales previas de las personas que dinamizan y participan de las acciones propuestas desde \#EA26 ya eran una referencia en el sector. Al haber logrado ser tendencia en las redes sociales han aumentado su protagonismo tanto en el propio sector como en sectores transversales $\mathrm{y}$ en las vertientes institucionales.

El equipo que dinamiza \#EA26 comparte una visión abierta y amplia de la Educación Ambiental, entendida como sensibilización, comunicación, participación y vinculada a los principios básicos y los objetivos que plantea "El Libro Blanco de la Educación Ambiental en España" y ahora, más que nunca, como una herramienta fundamental para afrontar la crisis climática.

Varias respuestas señalan que \#EA26 tiene características propias del ciberactivismo, tales como tener un discurso con el que cree poder cambiar las cosas, herramientas para generar consensos, capacidad de transmitir ideas en una red distribuida y lograr visibilidad a través de herramientas pensadas para que la gente, mediante pequeños gestos, pueda reconocerse en otros como ellos (empowering people).

Por otra parte, los debates \#EA26 han dado espacio de participación y se han valido de los denominados microinfluencers, personas que sin un elevado número de seguidores sí son influyentes en el nicho de la Educación Ambiental ayudando a la dispersión de sus líneas narrativas y argumentales.

Dado lo que las personas del equipo dinamizador aportan, parece evidente que \#EA26 tiene ante sí algunos retos de relevancia, como seguir siendo un movimiento referente y mantener el debate como estructura para continuar. Además, se pone de relieve seguir manteniendo la presencia, especialmente en las redes sociales y fuera de ellas, y pone de manifiesto la importancia de trabajar hacia una nueva Estrategia Estatal de Educación Ambiental.

Se manifiesta también la voluntad de llegar a un público más amplio y de seguir avanzando y evolucionando sin perder la esencia que ha llevado a muchas personas a sumarse a \#EA26, manteniendo el equilibrio entre la espontaneidad y frescura que la caracteriza y la relevancia que está adquiriendo.

Por lo tanto, el futuro de \#EA26 tras más de cinco años de existencia se plantea a medio plazo, con la consolidación del liderazgo en Twitter, la ampliación de su presencia en otras redes sociales, así como más allá del espacio virtual, como son los encuentros presenciales de \#EA26, el seguimiento del decálogo entregado a la Ministra de Transición Ecológica o la participación en la futura y necesaria Estrategia Estatal de Educación Ambiental. 
De las respuestas obtenidas y el conocimiento de la evolución de \#EA26 se podría deducir que uno de los retos más importantes a los que se enfrenta internamente es conjugar su exitosa fórmula de funcionamiento voluntaria (con distintos planteamientos y niveles de implicación) con dar respuesta al incremento de las demandas que recibe para aumentar su presencia y su incidencia social y política, en el apoyo a la Educación Ambiental o por el cambio ecosocial en el camino de la transición ecológica.

\section{Agradecimientos}

A todo el colectivo \#EA26 que ha participado de una forma u otra en que este movimiento esté en marcha.

\section{Referencias bibliográficas}

Alzamora, G., y Braga, C. (2014). Las redes sociales armas de protesta. Twitter y Facebook en las protestas de movimientos sociales en España y Brasil. En C. Ferré (Ed.), El uso de las redes sociales: ciudadanía, política y comunicación. La investigación en España y Brasil. (pp. 16-28) Bellaterra, España: Institut de la Comunicació, Universitat Autònoma de Barcelona.

Asociación de Ciencias Ambientales (CONAMA 10) (2010). Cómo usar las redes sociales para actuar en el medio ambiente. Recuperado de https://goo.gl/eu74vK.

Aznar Minguet, P., y Martínez Agut, M. P. (2013). La perspectiva de la sostenibilidad en la sociedad del conocimiento interconectado: gobernanza, educación, ética. Tesi, 14(3), 37-60.

Benayas, J., Marcén, C., Alba, D., y Gutiérrez, J. M. (2017). Educación para la sostenibilidad en España. Reflexiones y propuestas. Madrid, España: Fundación Alternativas y Red Española para el Desarrollo.

Breiting, S. (1985). Miljøundervisning - hvad er det? Pedagogisk Orientering, 5-6, 2635.

Brulle, R. (2010). From Environmental Campaigns to Advancing the Public Dialog: Environmental Communication for Civic Engagement. Environmental Communication, 4(1), 82-98

Cabero, J., y Llorente, M. C. (2005). Las TIC y la Educación Ambiental. Revista Latinoamericana de Tecnología Educativa, 4(2), 9-26.

Castillo Esparcia, A. (2014). Las salas de prensa virtuales en las organizaciones. El caso de las operadoras de telefonía móvil en España. En D. Fernández-Quijada y M. Ramos-Serrano (Eds.), Tecnologías de la persuasión. Uso de las TIC en publicidad y relaciones públicas. (pp. 89-113). Barcelona, España: Editorial UOC.

De la Osa Tomás, J. (2018). Prólogo. En D. Rodrigo-Cano, P. de Casas-Moreno, y P. Toboso (Eds.), Los medios de comunicación como difusores del cambio climático. (pp. 9-12). Sevilla, España: Egregius.

Fernández-Reyes, R. (2018). The communication of adaptation to Climate Change in spanish press. En R. Fernández-Reyes y D. Rodrigo Cano (Eds.), La comunicación de la mitigación. (pp. 135-175). Sevilla, España: Egregius. 
Ferreras, J., Calvo, S., y Rodrigo-Cano, D. (2019). La Red nos atrapa. Hacia una educación ambiental abierta y libre. En Javier Benayas y Carmelo Marcén (Eds.), Hacia una Educación para la Sostenibilidad (pp. 239-262). Madrid: Red Española para el Desarrollo Sostenible.

Fundación Telefónica (2017). Sociedad Digital en España 2017. Madrid, España: Ediciones Ariel.

García, J. E. (2002). Los problemas de la Educación Ambiental: ¿es posible una Educación Ambiental integradora? Revista Investigación en la Escuela, 46, 5-25.

Kaplún, M. (1998). Una pedagogía de la comunicación. Madrid, España: Ediciones de la Torre.

Kemp, S. (2018). Digital in 2018: world's internet users pass the 4 billion mark. We are social.com. Recuperado de https://goo.gl/4HFLxE.

León, B., y Bourk, M. (2018). Investigating Science-Related Online Video. En B. León, y M. Bourk (Eds.), Communicating Science and Technology Through Online Video. (pp. 1-15). Nueva York, EE.UU.: Routledge.

Meira Cartea, P. (2002). El cambio climático y la educación ambiental neoliberal (que también existe). Boletín Carpeta Informativa del CENEAM, 1-10. Recuperado de https://goo.gl/TfD23V.

Meira Cartea, P. (2013). Representaciones sociales del cambio climático en la sociedad española: una lectura para comunicadores. En R. Fernández-Reyes (Director), Mancinas-Chávez, R. (Coordinadora) (2013), Actas de las Jornadas Internacionales Medios de Comunicación y Cambio Climático (pp. 59-90). Sevilla, España: Fénix editora.

Meira, P., Barba, M., y Lorenzo, J. J. (2017). Crisis económica y profesionalización en el campo de la educación ambiental: comparativa 2007-2013 en Galicia. Educação $e$ Pesquisa, 43(4), 1127-1146. Epub 03 de abril de 2017. doi: http://dx.doi.org/10.1590/S1517-9702201703155092.

Ministerio de Agricultura, Pesca, Alimentación y Medio Ambiente (1999). Libro Blanco de la Educación Ambiental. Recuperado de https://goo.gl/3qRX36.

Navarro, M., Gutiérrez, D., Alcañiz, G., Martínez, R., Guitérrez, M., Ferreras, J., RodrigoCano, D., y Calvo, S. (Coords.) (2016). \#EA26. Un punto de encuentro para educadores y educadoras ambientales en las redes sociales. Carpeta CENEAM, julio-agosto. Recuperado de https://goo.gl/4t8tKZ.

Ojeda-Barceló, F., Gutiérrez-Pérez, J., y Perales-Palacios, F. J. (2009). ¿Qué herramientas proporcionan las tecnologías de la información y la comunicación a la educación ambiental? Revista Eureka sobre Enseñanza y Divulgación de las Ciencias, 6(3), 318-344.

Piñeiro, C., y Díaz-González, M. J. (2019). La educación ambiental en España desde las organizaciones sociales y los movimientos ciudadanos. En Javier Benayas y Carmelo Marcén (Eds.), Hacia una Educación para la Sostenibilidad (pp. 67-99). Madrid: Red Española para el Desarrollo Sostenible.

Revuelta, G., y Corchero, C. (2017). Perfiles generacionales en el consumo de información científica. En J. Lobera (Ed.), Percepción social de la ciencia y la 
tecnología. (pp. 180-205). Madrid, España: Fundación Española para la Ciencia y la Tecnología.

Rodrigo-Cano, D. (2018). La \#educomunicaciónambiental ante el reto de los límites del crecimiento del planeta. Triclab.es. Recuperado de https://triclab.es/educomunicacionambiental/.

Rodrigo-Cano, D., Picó, M. J., y Dimuro, G. (2019). Los Objetivos de Desarrollo Sostenible como marco para la acción y la intervención social y ambiental. Retos Revista de Ciencias de la Administración y Economía, 9(17), 25-36. https://doi.org/10.17163/ret.n17.2019.02

Ruiz Robles, R. (2015). Las redes sociales como herramienta para comunicar el mensaje ambiental. Carpeta CENEAM, Diciembre, 2015. Recuperado de https://goo.gl/LzB8tS.

Toboso, P. (2016). \#EA26: Análisis de la iniciativa educación ambiental en Twitter. Trabajo Fin de Máster. Recuperado de https://goo.gl/nddXZy.

Toboso, P., De Casas Moreno, P., y Rodrigo-Cano, D. (2018). \#EA26. Análisis de la iniciativa de educación ambiental en Twitter. En D. Rodrigo-Cano, P. de CasasMoreno, y P. Toboso (Eds.), Los medios de comunicación como difusores del cambio climático. (pp. 145-162). Sevilla, España: Egregius.

Vázquez Castillo, L. (2015). Adopción y uso de sitios web y Facebook por parte de ONG de medio ambiente en México. Virtualis, 11(1), 93-113. 